\title{
Comparison of displacement of polyvinyl chloride and silicone left- sided double-lumen tubes during lateral positioning
}

\author{
Nyeong Keon Kwon, Sung Mee Jung, Sang-Jin Park, and Yonghee Han \\ Department of Anesthesiology and Pain Medicine, Yeungnam University School of Medicine, Daegu, Korea
}

\begin{abstract}
Background: Compared to an equivalent sized polyvinyl chloride (PVC) double-lumen tube (DLT), a silicone DLT has a shorter endobronchial segment. The aim of this study was to compare the incidence of clinically significant displacement of left-sided PVC and silicone DLTs after a positional change to a lateral position from a supine position and determine its effect on the need for DLT repositioning for successful lung separation in patients undergoing thoracic surgery.

Methods: One hundred eight adult patients requiring one-lung ventilation were randomly divided into group P (PVC DLT, $n=54$ ) and group S (Silicone DLT, $n=54$ ). The position of the DLT was observed before and after lateral positioning to assess the effect of the position change. The incidence of clinically significant displacement $(>10 \mathrm{~mm})$ of DLT was compared between the groups.

Results: DLTs were clinically significantly displaced in group P $(17 / 48,35.4 \%)$ and group S $(18 / 52,34.6 \%)$ after lateral positioning $(\mathrm{P}=0.933)$. A proximal displacement (31.3\% [group $\mathrm{P}$ ] and $25.0 \%$ [group $\mathrm{S}$ ]) was more common than distal displacement (4.2\% [group $\mathrm{P}$ ] and $9.6 \%$ [group $\mathrm{S}$ ]), with no significant intergroup difference $(\mathrm{P}=0.494)$. After lateral positioning, critical malposition of DLT with bronchial herniation to the right main bronchus was $8.3 \%$ (group P) and $7.9 \%$ (group S, P = 0.906).

Conclusions: Left-sided PVC and silicone DLTs produced comparable incidences of clinically significant displacement and, consequently, required similar rates of repositioning for successful lung separation after lateral positioning.
\end{abstract}

Keywords: Bronchoscopy; Displacement; Intratracheal intubation; One-lung ventilation; Posture; Thoracic surgical procedure.

Corresponding author: Sung Mee Jung, M.D., Ph.D.

Department of Anesthesiology and Pain Medicine, Yeungnam University School of Medicine, 170 Hyeonchung-ro, Nam-gu, Daegu 42415, Korea

Tel: 82-53-620-3368, Fax: 82-53-625-5275

Email: applejsm@gmail.com

ORCID: https://orcid.org/0000-0001-5602-9011

It was presented at ANESTHESIOLOGY 2017 Annual Meeting of The American Society of Anesthesiologists (ASA), October 2017, Boston Convention and Exhibition Center, Boston, USA

Received: January 12, 2018.

Revised: March 13, 2018 (1st); April 6, 2018 (2nd); May 1, 2018 (3rd). Accepted: May 14, 2018.

Korean J Anesthesiol 2019 February 72(1): 32-38 https://doi.org/10.4097/kja.d.18.00012

\section{Introduction}

The correct positioning of the double-lumen endobronchial tube (DLT) is essential for lung separation with the collapse of the operating lung and ventilation of the non-operating lung (one-lung ventilation; OLV) in patients undergoing thoracic surgery. A left-sided DLT is usually preferred in most thoracic surgeries because of a greater risk of upper lobe obstruction with a right-sided DLT [1]. However, even well-positioned DLTs with an appropriately inflated endobronchial cuff are known to get displaced during a change in patient position and surgical manipulation of the bronchus [2-5]. Left-sided polyvinyl chloride (PVC) DLTs are recommended to have the upper edge of the en-

(c) This is an open-access article distributed under the terms of the Creative Commons Attribution Non-Commercial License (http://creativecommons.org/ licenses/by-nc/4.0/), which permits unrestricted non-commercial use, distribution, and reproduction in any medium, provided the original work is properly cited. 
dobronchial cuff be seen at least $5 \mathrm{~mm}$ below the tracheal carina in the left main bronchus by fiberoptic bronchoscopy $[6,7]$.

Presently, Robertshaw-design DLTs, made of PVC and silicone materials, are commonly used in clinical practice. Compared to PVC DLT, silicone DLT has a wire-reinforced endobronchial lumen to prevent obstruction of bronchus from mediastinal compression when the tube is positioned in the dependent position. Compared to an equivalent-sized PVC DLT, the shorter endobronchial segment of the silicone DLT due to a narrower endobronchial cuff and a shorter, unbeveled, distal endobronchial tip can reduce the risk of left upper lobe bronchus obstruction but may augment the risk of dislodgement from the left main bronchus (Fig. 1). Lee et al. [8] reported that the endobronchial cuff of the silicone DLT (Silbroncho ${ }^{\circledR}$, Fuji Systems Co., Japan) should be positioned $20 \mathrm{~mm}$ below the tracheal carina rather than $5 \mathrm{~mm}$ to provide the same margin of safety for obstruction of left upper lobe bronchus as PVC DLT as well as cuff herniation into the tracheal carina. However, it is unclear if the clinical recommendations for correct DLT positioning based on the use of the PVC DLT are applicable to the use of silicone DLTs for effective lung separation with a change in patient position and thoracic surgical manipulation.

Therefore, the aim of this study was to compare the incidences of clinically significant displacement of left-sided PVC and silicone DLTs using a fiberoptic bronchoscope (FOB) and to determine its effect on the need for DLT repositioning for successful lung separation when the patient was moved from the supine to the lateral decubitus position when undergoing thoracic surgery.

\section{Materials and Methods}

This prospective, randomized single-blind study was approved by the Institutional Review Board of Yeungnam University Hospital and registered with ClinicalTrials.gov (NCT02691468). After written informed consent was obtained, 108 adult patients, aged 18 to 75 years, American Society of Anesthesiologists physical status I-III, scheduled for elective thoracic surgery with the use of left-sided DLT were enrolled for this study. Patients with absolute indication for a right-sided DLT insertion or single-lumen tube with a bronchial blocker, emergency surgery, surgery requiring a sternotomy, an intraluminal lesion in the left bronchus, or a distorted tracheobronchial tree anatomy due to an intraluminal tumor or extrinsic mass on the preoperative chest radiograph were excluded from the study. A total of one hundred and eight patients were enrolled in this study (Fig. 2). One hundred patients ( $\mathrm{n}=48$ in group $\mathrm{P}$ and $\mathrm{n}=$ 52 in group S) were included in the final analysis after excluding eight participants ( $n=6$ in group $P$ and $n=2$ in group $S$ ).

Before surgery, the length of the left main bronchus was
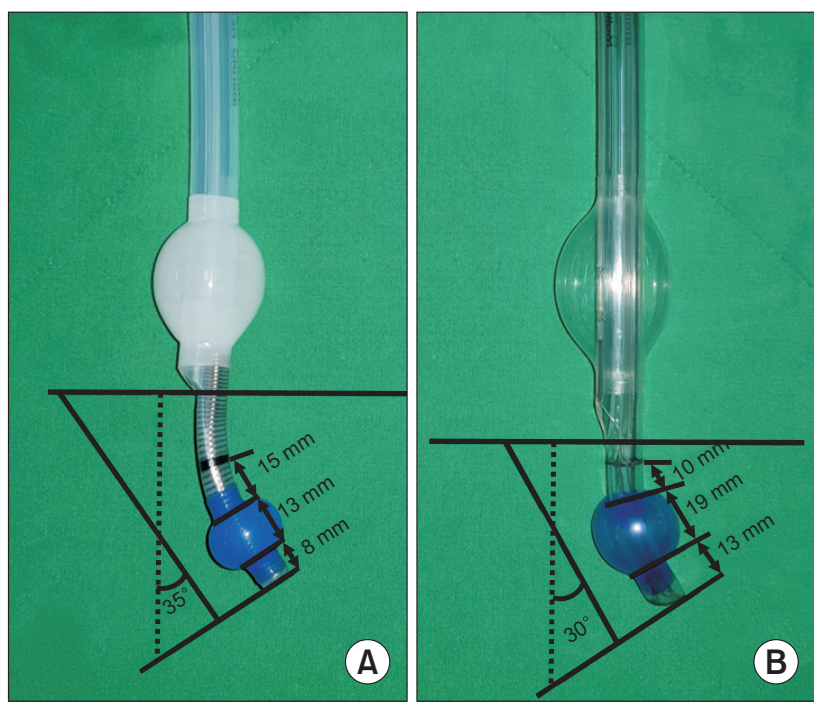

Fig. 1. (A) A 37 Fr silicone (Human Bronch ${ }^{\circledR}$ ) double-lumen tube, (B) A 37 Fr polyvinyl chloride (Broncho-Cath ${ }^{\mathrm{TM}}$ ) double-lumen tube.

measured as the distance from the tracheal bifurcation point to the bronchial bifurcation point between the left upper and lower lobe bronchus on a two-dimensional chest computed tomography (CT) imaging. The anteroposterior and transverse diameters of the left main bronchus were measured $2 \mathrm{~cm}$ below the tracheal carina, where the bronchial cuff of left-sided DLT is conventionally placed. The right and left bronchial angles were defined as the deviation of the central axis of each bronchus from a vertical line passing through the inferior point of the tracheal bifurcation.

All patients received glycopyrrolate $0.2 \mathrm{mg}$ intramuscularly to decrease secretion $30 \mathrm{~min}$ before anesthesia. After arriving in the preoperative holding area, they were randomly allocated into two groups using a computer-generated random assignment scheme; group P (PVC DLT, Broncho-Cath ${ }^{\mathrm{TM}}$, Covidien, USA) and group S (Silicone DLT, Human Broncho ${ }^{\circledR}$, Insung Medical Co., Korea) (Fig. 1). Anesthesia was induced and maintained with total intravenous anesthesia using a target-controlled infusion of propofol and remifentanil under the monitoring of blood pressure, oxygen saturation, electrocardiogram, and bispectral index for measurement of hypnotic depth. After inducing adequate neuromuscular relaxation with the administration of $0.8 \mathrm{mg} / \mathrm{kg}$ of rocuronium intravenously, a left-sided DLT was inserted into the trachea until the depth would be approximately $12+($ height/10) $\mathrm{cm}$ at the teeth by direct laryngoscopy in the supine position [9]. The endobronchial cuff was inflated to the minimal volume that seals the air leak during OLV using an airfilled syringe. The position of the DLT was initially determined by auscultation of breathing sounds with clamping the tracheal lumen and bronchial lumen alternately. Pressure-controlled ventilation was performed. The inspiratory fraction of oxygen and 


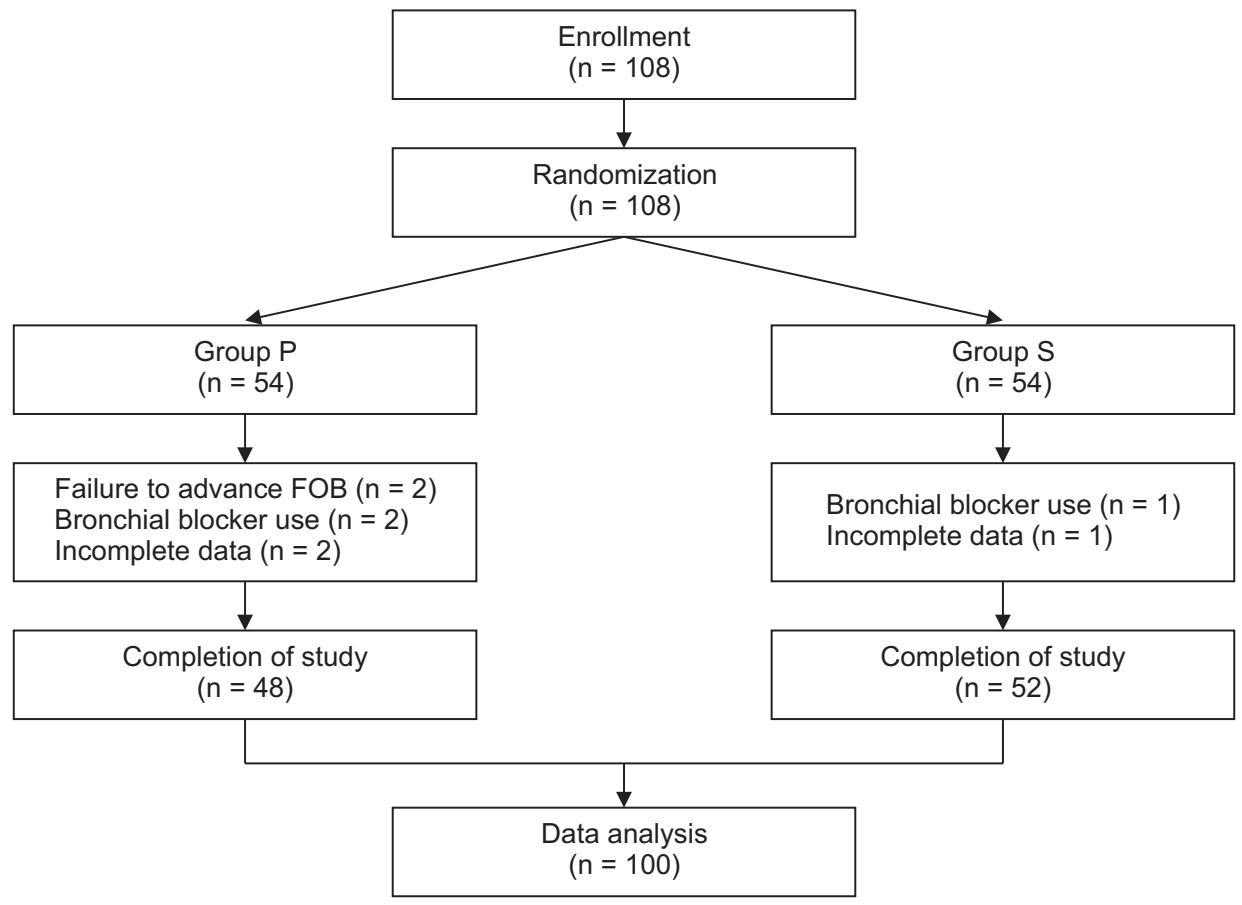

Fig. 2. Flow Chart. Group P: polyvinyl chloride double-lumen tube, Group S: silicone double-lumen tube. FOB: fiberoptic bronchoscope. delivered tidal volume was closely monitored to maintain $\mathrm{SaO}_{2}$ greater than 95\%. Respiratory rate was set to maintain $\mathrm{PaCO}_{2}$ between 35 and $45 \mathrm{mmHg}$ during OLV and two-lung ventilation. Lateral positioning for thoracic surgery was performed with the endobronchial cuff inflated by holding the DLT at the level of the incisors and keeping the patient's head and neck immobile in the neutral position.

A single independent investigator performed bronchoscopy through tracheal and bronchial lumens using a FOB (Olympus LF-2, Olympus Optical Co., Japan). The initial position of the DLT was observed after blind insertion of the tube in the supine position. The correct position of the DLT was defined as both tracheal and bronchial carinas were clearly visualized and the upper edge of the endobronchial cuff was $5 \mathrm{~mm}$ below the tracheal carina in the left main bronchus [6] and was achieved using FOB guidance. When a FOB was introduced into the tracheal lumen until the upper edge of the bronchial cuff was in line with the tracheal carina, a piece of tape was placed on the external surface of the bronchoscope at the point where it entered the self-sealing diaphragm in the elbow connector. And then the DLT was advanced $5 \mathrm{~mm}$ into the left main bronchus from the external marking on the bronchoscope so that the upper edge of the endobronchial cuff was positioned to be 5 $\mathrm{mm}$ below the tracheal carina in the left main bronchus. The position of the DLT was observed at four time-points; (1) after correctly repositioning the tube in the supine position, (2) after change of position from the supine to the lateral decubitus, (3) at start of surgery after correctly repositioning the tube in the lateral decubitus position, and (4) at the end of surgery in the lateral decubitus position. The tracheal distance was defined as the distance between the distal tip of the tracheal lumen and tracheal carina whereas the bronchial distance was defined as that between the bronchial carina and distal tip of the endobronchial lumen. The displacement of DLT was determined by changes in tracheal and bronchial distances, obtained by subtracting supine measurements from lateral measurements and subtracting measurements at the start of surgery from measurements at the end of surgery, respectively. Clinically significant displacement was defined when the DLT was deviated by more than $10 \mathrm{~mm}$ from the initial correct position, regardless of the direction of displacement [10]. A critical malposition requiring immediate reposition of DLT for successful OLV was defined as obstruction of the left upper or lower lobe bronchus by the distal tip of the endobronchial lumen or the intratracheal dislocation of more than half of the endobronchial cuff. The primary endpoint of this study was the incidence of clinically significant displacement of the left-sided PVC and silicone DLTs during a change of patient position from supine to lateral decubitus. The secondary endpoint of this study was the incidence of critical malposition of DLTs requiring reposition for successful OLV during position change.

The sample size was calculated using $\mathrm{G}^{\star}$ Power ver. 3.1.5. It was ascertained that 48 patients were required in each group to detect an intergroup difference of $30 \%$ in the incidence of clinically significant DLT displacement during positional change with $\alpha$ of 0.05 and a power of $80 \%$. Fifty-four participants per 
Table 1. Demographic, Anesthetic, and Surgical Characteristics of the Patients

\begin{tabular}{|c|c|c|c|}
\hline Variable & Group P $(\mathrm{n}=48)$ & Group S $(n=52)$ & $P$ value \\
\hline Age (yr) & $56.7 \pm 16.1$ & $57.8 \pm 14.9$ & 0.704 \\
\hline $\operatorname{Sex}(M / F)$ & $34 / 14$ & $35 / 17$ & 0.703 \\
\hline Weight $(\mathrm{kg})$ & $64.3 \pm 10.3$ & $60.9 \pm 8.7$ & 0.074 \\
\hline Height $(\mathrm{cm})$ & $165.5 \pm 9.3$ & $163.7 \pm 7.6$ & 0.290 \\
\hline ASA physical status (I/II/III) & $13 / 28 / 7$ & $10 / 37 / 5$ & 0.404 \\
\hline \multicolumn{4}{|l|}{ Left main bronchus } \\
\hline Length (mm) & $49.2 \pm 5.3$ & $47.9 \pm 5.0$ & 0.209 \\
\hline \multicolumn{4}{|l|}{ Diameter $(\mathrm{mm})$} \\
\hline Anteroposterior & $13.4 \pm 2.0$ & $13.2 \pm 2.0$ & 0.635 \\
\hline Transverse & $14.4 \pm 2.1$ & $14.6 \pm 2.0$ & 0.750 \\
\hline \multicolumn{4}{|l|}{ Bronchial angle $\left({ }^{\circ}\right)$} \\
\hline Right & $37.3 \pm 8.5$ & $39.2 \pm 9.6$ & 0.311 \\
\hline Left & $41.2 \pm 7.1$ & $41.8 \pm 7.0$ & 0.721 \\
\hline Size of double-lumen tube (Fr) & & & 0.703 \\
\hline 35 & $14(29.2 \%)$ & $17(32.7 \%)$ & \\
\hline 37 & $34(70.8 \%)$ & $35(67.3 \%)$ & \\
\hline Side of surgery & & & 0.733 \\
\hline Right & $27(56.3 \%)$ & $31(59.6 \%)$ & \\
\hline Left & $21(43.8 \%)$ & $21(40.4 \%)$ & \\
\hline Type of surgery & & & 0.061 \\
\hline Thoracotomy & $32(66.7 \%)$ & $25(48.1 \%)$ & \\
\hline Thoracoscopy & $16(33.3 \%)$ & $27(51.9 \%)$ & \\
\hline Duration of anesthesia (min) & $257.9 \pm 97.8$ & $227.8 \pm 93.2$ & 0.118 \\
\hline Duration of operation (min) & $164.2 \pm 73.9$ & $151.0 \pm 86.9$ & 0.417 \\
\hline
\end{tabular}

Data are presented as mean \pm SD or number of patients (\%). Group P: polyvinyl chloride double-lumen tube, Group S: silicone double-lumen tube.

Table 2. Lengths and Incidences of Tracheal and Left Main Bronchial Displacement

\begin{tabular}{lccc}
\hline & Group P $(\mathrm{n}=48)$ & Group S (n=52) & P value \\
\hline Lateral positioning & & & \\
Tracheal displacement $(\mathrm{mm})$ & $6.4 \pm 10.0(-10.6,23.0)$ & $3.1 \pm 10.4(-18.8,19.8)$ & 0.104 \\
Bronchial displacement (mm) & $4.1 \pm 9.4(-12.6,21.5)$ & $6.2 \pm 10.1(-16.6,25.4)$ & 0.289 \\
Clinically significant displacement & $17(35.4 \%)$ & $18(34.6 \%)$ & 0.933 \\
Critical malposition* & $4(8.3 \%)$ & $4(7.9 \%)$ & 0.906 \\
Surgical manipulation & & & 0.070 \\
Tracheal displacement (mm) & $1.3 \pm 8.3(-15.2,18.3)$ & $-2.0 \pm 9.0(-15.2,13.8)$ & 0.499 \\
Bronchial displacement (mm) & $-0.9 \pm 5.1(-9.0,9.0)$ & $-2.0 \pm 9.4(-19.8,14.0)$ & 0.285 \\
Clinically significant displacement & $10(21.7 \%)$ & $16(31.4 \%)$ & \\
Critical malposition* & 0 & 0 &
\end{tabular}

Data are presented as mean \pm SD (range) or the number of patients (\%). The displacement of the double-lumen tube was determined by changes in tracheal and bronchial distances, obtained by subtracting supine measurements from lateral measurements and subtracting measurements at the start of surgery from measurements at the end of surgery, respectively. The direction of displacement is presented as positive values for proximal movement and negative values for distal movement, respectively. Clinically significant displacement was defined when the double-lumen tube was deviated by more than $10 \mathrm{~mm}$ from the initial correct position, regardless of the direction of displacement. *Requiring reposition of the double-lumen tube for successful one-lung ventilation. Group P: polyvinyl chloride double-lumen tube, Group S: silicone double-lumen tube.

group were recruited to compensate for a $10 \%$ dropout rate. Statistical analysis was performed using SPSS 22.0 for Windows (IBM Corp., USA). Categorical data were presented as the number of patients (percentages) and analyzed using Chi-square test or Fischer's exact test as appropriate. Continuous data were presented as the mean \pm SD for normally distributed variables and analyzed using the independent Student's $t$-test and the Mann-Whitney $U$ test, respectively. A $\mathrm{P}$ value $<0.05$ was considered statistically significant. 


\section{Results}

The demographic, anesthetic, and surgical data were comparable between the two groups (Table 1). All men were intubated with 37 Fr DLTs and all women were intubated with 35 Fr DLTs. After initial blind intubation of DLT in the supine position, auscultation of breathing sounds detected unsatisfactory lung separation in $6(12.5 \%)$ in group $\mathrm{P}$ and 5 (9.6\%) in group $\mathrm{S}(\mathrm{P}$ $=0.645)$. However, DLTs were observed to be positioned incorrectly in 15 patients (31.3\%) in group $\mathrm{P}$ and 10 (19.2\%) in group $\mathrm{S}$ by fiberoptic bronchoscopy $(\mathrm{P}=0.166)$. Incidences of bulging of the endobronchial cuff into the tracheal carina $(29.2 \%$ in group P and $15.4 \%$ in group S) and obstruction of the left upper bronchus by the endobronchial cuff (2.1\% in group P and 3.8\% in group $\mathrm{S}$ ) were comparable between the two groups $(\mathrm{P}=0.338)$. The lengths of tracheal and left bronchial displacement occurring when the patients were shifted to the lateral decubitus from the supine position were comparable between the two groups (Table 2). Clinically significant displacement of DLTs (> $10 \mathrm{~mm}$ ) from the correct position were observed in $35.4 \%$ in group $\mathrm{P}$ and $34.6 \%$ in group $\mathrm{S}$, on bronchoscopic inspection $(\mathrm{P}=0.933$, Table 2). A proximal displacement (31.3\% in group $\mathrm{P}$ and $25.0 \%$ in group $\mathrm{S}$ ) was more common than a distal one (4.2\% in group $\mathrm{P}$ and $9.6 \%$ in group $\mathrm{S}$ ), which was comparable between the two groups ( $\mathrm{P}=0.494$, Fig. 3). After lateral positioning, critical malposition of DLT with endobronchial cuff herniation into the trachea and right main bronchus was observed in 8 patients $(8.3 \%$ in group $\mathrm{P}$ and $7.9 \%$ in group $\mathrm{S}, \mathrm{P}=0.906$, Table 2).

Surgical manipulation in lateral decubitus position resulted in a clinically significant displacement of DLT in $21.7 \%$ in group $\mathrm{P}$ and $31.4 \%$ in group $\mathrm{S}(\mathrm{P}=0.285$, Table 2$)$. The lengths of the intraoperative tracheal and left bronchial displacements in lateral decubitus position were comparable across the two groups

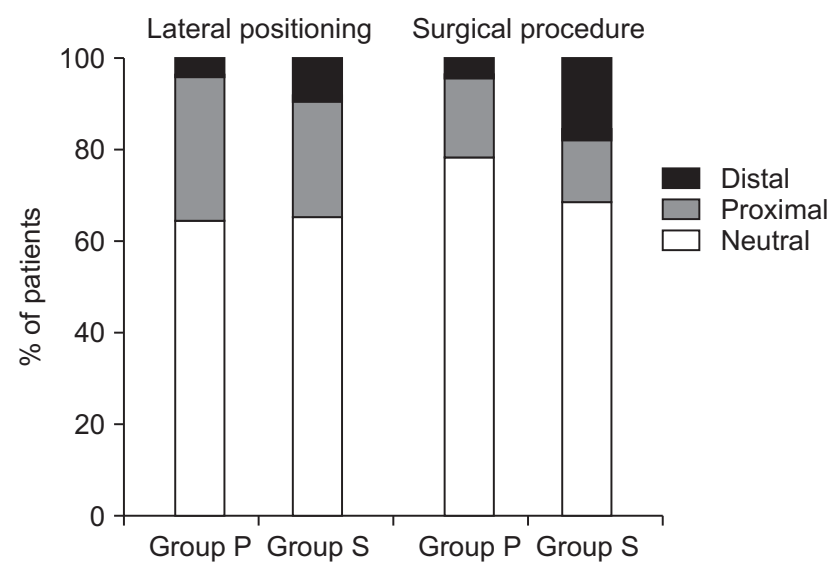

Fig. 3. Direction of clinically significant displacement of the left-sided double-lumen tube. Group P: polyvinyl chloride double-lumen tube, Group S: silicone double-lumen tube.
(Table 2). Surgical manipulation displaced the DLT comparably in the distal (4.3\% in group P and $17.6 \%$ in group S) and proxi$\mathrm{mal}$ (17.4\% in group $\mathrm{P}$ and $13.7 \%$ in group S) directions in both groups ( $\mathrm{P}=0.117$, Fig. 3 ). However, no patient required DLT repositioning due to critical malposition during surgery.

In both groups, lateral positioning induced greater length of displacement than surgical manipulation in both tracheal and bronchial distances (tracheal displacement; 95\% CI of the mean difference, 1.2-9.6, $\mathrm{P}=0.014$ in group $\mathrm{P}$ and $95 \% \mathrm{CI}$ of the mean difference, $0.4-9.2, \mathrm{P}=0.031$ in group $\mathrm{S}$ and bronchial displacement; 95\% CI of the mean difference, 0.4-9.2, $\mathrm{P}=0.031$ in group $\mathrm{P}$ and $95 \% \mathrm{CI}$ of the mean difference, $3.8-12.3, \mathrm{P}<$ 0.001 in group S, Table 2).

\section{Discussion}

In the present study, left-sided PVC and silicone double-lumen tubes presented with comparable incidences of clinically significant displacement and, consequently, required similar rates of tube repositioning for successful lung separation after turning patients from the supine to the lateral decubitus position. When the endobronchial cuff was positioned correctly 5 $\mathrm{mm}$ below the tracheal carina in the supine position [6], the PVC and silicone DLTs had comparable tracheal and bronchial displacement during the change in patient position. The tubes were displaced greater than $10 \mathrm{~mm}$ in $35.4 \%$ of patients using PVC DLT and in $34.6 \%$ of patients using silicone DLT after lateral positioning. The direction of displacement was predominantly proximal and all critical malpositions requiring immediate DLT repositioning resulted from endobronchial cuff herniation to the trachea and right main bronchus in both PVC and silicone DLTs.

After the introduction of PVC DLTs in the 1980s [11], a silicone DLT was constructed with a wire-reinforced endobronchial lumen to prevent obstruction and kinking from mediastinal compression when the tube is positioned in the dependent lung. Furthermore, the silicone DLT has a narrower endobronchial cuff (13 mm versus $19 \mathrm{~mm}$ ) and a shorter endobronchial lumen beyond the cuff ( $8 \mathrm{~mm}$ versus $13 \mathrm{~mm}$ ) compared to an equivalent sized PVC DLT. This shortened endobronchial cuff-tip length of the silicone DLT can provide a greater margin of safety for obstruction of upper lobe bronchus in the left main bronchus. However, the narrower endobronchial cuff of silicone DLT may present a higher risk of cuff herniation into the tracheal carina even with a displacement equivalent to the PVC DLT after lateral positioning. A clinical study had been performed to investigate whether the silicone DLT with a shorter endobronchial cuff-tip length compared to PVC DLT might need different guidelines for positioning of the tube for successful lung separation in patients undergoing thoracic surgery. The more deeply 
positioned silicone DLTs may have an increased margin of safety for proximal displacement and, consequently, a diminished risk of endobronchial cuff herniation into the right main bronchus after lateral positioning compared to an equivalent sized PVC DLT. However, because the authors did not determine the length and direction of silicone DLT displacement during a change of patient position, it was not determined whether the positioning of PVC and silicone DLTs in the supine position should be different for successful lung separation.

The margin of safety in positioning left-sided DLT is the length of the left main bronchus over which the endobronchial segment of the tube can be moved in the bronchus without obstructing the airway [12]. It is the length of the left main bronchus minus the distance from the upper edge of the endobronchial cuff to the tip of the endobronchial lumen (cuff-tip length). Generally, the most acceptable positioning of DLT is considered when, proximally, the upper edge of the endobronchial cuff is just below the carina and, distally, the tip of the endobronchial lumen is at the proximal edge of the left upper lobe bronchial orifice. If the displacement exceeds this acceptable limit, the DLT should be repositioned for successful lung separation. Desiderio et al. [2] reported that the endobronchial cuff should be placed at least $10 \mathrm{~mm}$ below the tracheal carina inside the left main bronchus in the supine position because of the tendency for a downward carinal shift and a $10 \mathrm{~mm}$ upward movement of the DLT during lateral positioning. They also suggested that the FOB should be kept available during the procedure as an additional shifting of the DLT can occur with surgical manipulation.

In the present study, when the endobronchial cuff was positioned $5 \mathrm{~mm}$ below the tracheal carina in the supine position, nearly one-third of both types of DLT was displaced greater than $10 \mathrm{~mm}$ predominantly in the proximal direction after lateral positioning, which would increase the margin of safety for obstruction of the left upper lobe bronchus but simultaneously reduce the margin of safety for obstruction of the right main bronchus. When patients were turned from the supine to the lateral decubitus position, all critical malposition cases in both left-sided DLTs involved endobronchial cuff herniation into the right main bronchus in our patients. Although to a lesser extent than that produced by the change in patient position, surgical manipulation also produced a clinically significant displacement in $20-30 \%$ of the patients. Due to surgical traction in addition to lateral positioning, the tube may progressively migrate above the tracheal carina and obstruct the trachea and right main bronchus, especially in the proximal displacement. On the other hand, the mean length of left main bronchus measured from the chest CT was $49.5 \mathrm{~mm}$ in male in the present study; when the endobronchial cuff was positioned $5 \mathrm{~mm}$ below the tracheal carina, the distance from the distal tip of the endobronchial lumen to the left bronchial carina would be approximately $13 \mathrm{~mm}$ for
PVC DLTs and $24 \mathrm{~mm}$ for silicone DLTs in the supine position. This could allow for maintenance of an adequate margin of safety for obstruction of the left upper lobe bronchus, even with a displacement in the distal direction during lateral positioning or surgical manipulation in our patients. However, in this study, the shortest length of left main bronchus was $43.3 \mathrm{~mm}$, which implies that the margin of safety for left upper lobe orifice in PVC DLT and silicone DLT is $1.3 \mathrm{~mm}$ and $12.3 \mathrm{~mm}$, respectively, when the endobronchial cuffs of both DLTs are positioned 10 $\mathrm{mm}$ below the tracheal carina as Desiderio et al. [2] suggested. In spite of the proximal displacement tendency of DLT after lateral positioning and subsequent additional displacement during surgical manipulation, positioning the upper edge of the endobronchial cuff of PVC DLT more than $5 \mathrm{~mm}$ below the tracheal carina in the supine position should be cautious because of an inadequate margin of safety for left upper lobe obstruction. By contrast, positioning the upper edge of the endobronchial cuff of silicone DLT $10 \mathrm{~mm}$ below the tracheal carina in the supine position would provide an adequate margin of safety for both left upper lobe obstruction and endobronchial cuff herniation to the contralateral bronchus after lateral positioning.

This study has some limitations. First, we correctly adjusted DLT positioning before lateral positioning in the supine position and before the start of the surgery in the lateral decubitus position, respectively. The position change and surgical manipulation may have a combined, inter-related influence on the tracheal and bronchial cuff displacement. Therefore, the simple sum of displacement during position change and during surgical manipulation after corrective adjustment of DLT, respectively, may be different from the consecutive displacement of DLT throughout position change and surgical manipulation. Second, we did not monitor the pressure and volume of air injected into the bronchial cuff. Inappropriate cuff volume may influence DLT displacement during a change of position and surgical manipulation [2].

In conclusion, both left-sided PVC and silicone DLTs produced similar incidences of clinically significant displacement and repositioning for successful OLV after turning the patient from the supine to the lateral decubitus position for thoracic surgery.

\section{ORCID}

Nyeong Keon Kwon, https://orcid.org/0000-0003-0493-2203

Sung Mee Jung, https://orcid.org/0000-0001-5602-9011

Sang-Jin Park, https://orcid.org/0000-0002-4838-2664

Yonghee Han, https://orcid.org/0000-0002-5633-4099 


\section{References}

1. Brodsky JB, Lemmens HJ. Left double-lumen tubes: clinical experience with 1,170 patients. J Cardiothorac Vasc Anesth 2003; 17: 289-98.

2. Desiderio DP, Burt M, Kolker AC, Fischer ME, Reinsel R, Wilson RS. The effects of endobronchial cuff inflation on double-lumen endobronchial tube movement after lateral decubitus positioning. J Cardiothorac Vasc Anesth 1997; 11: 595-8.

3. Yoon TG, Chang HW, Ryu HG, Kwon TD, Bahk JH. Use of a neck brace minimizes double-lumen tube displacement during patient positioning. Can J Anaesth 2005; 52: 413-7.

4. Maruyama D, Chaki T, Omote M, Hirata N, Yamauchi M, Yamakage M. Movements of the double-lumen endotracheal tube due to lateral position with head rotation and tube fixation: a Thiel-embalmed cadaver study. Surg Radiol Anat 2015; 37: 841-4.

5. Pfitzner J, Stevens HJ, Lance DG. Identifying imminent displacement of a double-lumen tube caused by surgical traction at the pulmonary hilum. J Cardiothorac Vasc Anesth 2007; 21: 776-7.

6. Slinger PD, Campos JH. Anesthesia for thoracic surgery. In: Miller's Anesthesia. 8th ed. Edited by Miller RD: Philadelphia, Churchill Livingstone/Elsevier. 2015, pp 1942-2006.

7. Campos JH. Update on tracheobronchial anatomy and flexible fiberoptic bronchoscopy in thoracic anesthesia. Curr Opin Anaesthesiol 2009; 22: 4-10.

8. Lee JS, Kil TY, Chung JY. Comparison of a silicon double-lumen endobronchial tube (Silbroncho ${ }^{\circledR}$ ) with a polyvinyl chloride tube (Broncho-Cath $^{\circledR}$ ) in right-side thoracic surgery. Korean J Anesthesiol 2005; 48: 509-13.

9. Bahk JH, Oh YS. Prediction of double-lumen tracheal tube depth. J Cardiothorac Vasc Anesth 1999; 13: 370-1.

10. Inoue S, Nishimine N, Kitaguchi K, Furuya H, Taniguchi S. Double lumen tube location predicts tube malposition and hypoxaemia during one lung ventilation. Br J Anaesth 2004; 92: 195-201.

11. Burton NA, Watson DC, Brodsky JB, Mark JB. Advantages of a new polyvinyl chloride double-lumen tube in thoracic surgery. Ann Thorac Surg 1983; 36: 78-84.

12. Benumof JL, Partridge BL, Salvatierra C, Keating J. Margin of safety in positioning modern double-lumen endotracheal tubes. Anesthesiology 1987; 67: 729-38. 